\title{
Self-Healing Ring-Based Time-Sharing Passive Optical Networks
}

\author{
Chien-Hung Yeh and Sien Chi
}

\begin{abstract}
A new ring-based time-shared passive optical network (PON), with a self-healing function to prevent the occurrence of fiber-fault, has been proposed and investigated experimentally. By using the new optical line termination and single fiber path architecture, the proposed self-healing ring topology PON can be revived promptly under single fiber failure. The system performances of the proposed access network are also measured and discussed.
\end{abstract}

Index Terms-Passive optical network (PON), protection, ring.

\section{INTRODUCTION}

F IBER-TO-THE- $x$ (FTTx) deployments have taken precedent with several field trials and deployments implemented in different parts of the world in the last few years. It means that optical fiber access networks would provide the broadband bandwidth for both downstream and upstream traffic. Therefore, the time-division-multiplexing passive optical network (TDM-PON) is one of the choices for next-generation FTTx. Recently, TDM-PONs have been thoroughly explored and standardized and they are the first point-to-multipoint solutions moving into the filed. Some commercial products in the PON standards already exist [1]-[3]. In general, the architecture of PONs have three basic topologies, which are the bus-, tree- and ring-structure, respectively. The point-to-multipoint connectivity between the optical line termination (OLT) and multiple optical network units (ONUs) is obtained using a passive branching device at the remote node. The traffic link from an OLT to an ONU is called "downstream" (point-to-multipoint) and traffic from an ONU to the OLT is called "upstream" (multipoint-to-point). Typically, a 1310-nm wavelength for the upstream transmission and 1490-nm wavelength for the downstream transmission are used in PONs. When a fiber link from the OLT to the ONU is broken, the affected ONUs will become unreachable to OLT. Therefore, some fiber protection methods in PONs have been implemented [4], [5]. However, there has not been much discussion of the protection techniques in the ring topology PONs [5].

Manuscript received November 20, 2006; revised April 14, 2007. This work was supported in part by the National Science Council (NSC) of Taiwan (R.O.C.) under Grant NSC 95-2221-E-155-059 and Grant NSC 95-2221-E-155-072.

C.-H. Yeh is with the Information and Communications Research Laboratories, Industrial Technology Research Institute, Chutung, Hsinchu 310-40, Taiwan, R.O.C. (e-mail: depew @itri.org.tw).

S. Chi is with the Department of Photonics and Institute of Electro-Optical Engineering, National Chiao Tung University, Hsinchu 300-10, Taiwan, R.O.C., and also with the Department of Electrical Engineering, Yuan Ze University, Chungli 320-03, Taiwan, R.O.C.

Color versions of one or more of the figures in this letter are available online at http://ieeexplore.ieee.org.

Digital Object Identifier 10.1109/LPT.2007.900155

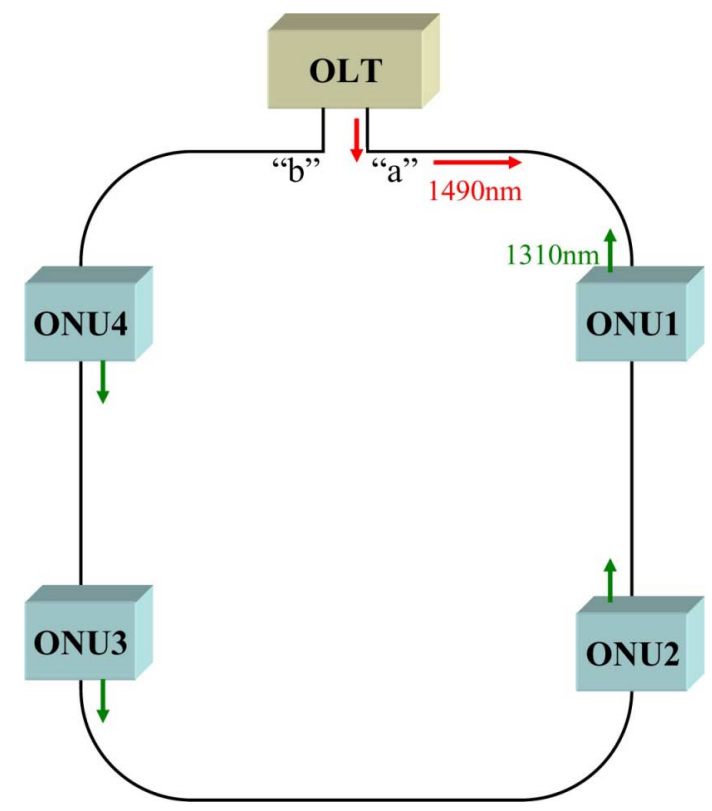

Fig. 1. Data traffic of proposed self-protecting ring-based PON with four ONUs in normal state.

In this letter, we propose and demonstrate a novel self-protecting architecture for a ring-based PON to prevent fiber failure in single-path. Moreover, the data traffic performances have also been measured and discussed in this ring-based access network.

\section{Proposed ARCHITECTURE}

When a conventional ring-based PON system has $n$ ONUs and a fiber fault occurs between $\mathrm{ONU}_{2}$ and $\mathrm{ONU}_{3}$, the downstream signals will be unreachable behind the fault point. That is to say, the OLT cannot link the upstream signals from $\mathrm{ONU}_{3}$ to $\mathrm{ONU}_{n}$. At this time, the PON system must have the self-healing function for data traffic to overcome the fiber fault problem. To achieve desired network survivability, the different protection schemes are recommended. The post protection method with double transceivers [or called line termination (LT)] at both ends and two individual fiber paths for the ring-based and tree-based TDM-PONs have also been reported [2], [4]. Furthermore, the two paths will increase the cost of building fiber and also need to add the other passive components. An additional transceiver in OLT and ONU also increases the cost in PONs [2], [5]. To improve these drawbacks as mentioned before, we propose and investigate a new ring-based $\mathrm{PON}$ with self-healing function only by one fiber path, as shown in Fig. 1. Moreover, we assume the proposed self-healing architecture system having four ONUs for data traffic, as shown in Fig. 1. 


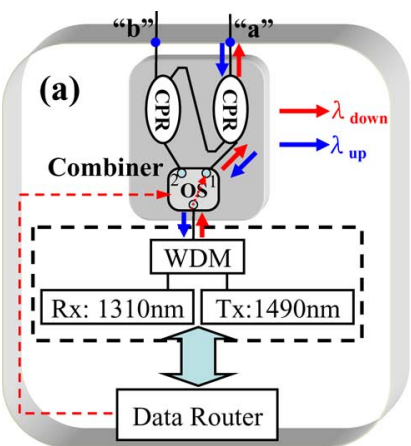

OLT

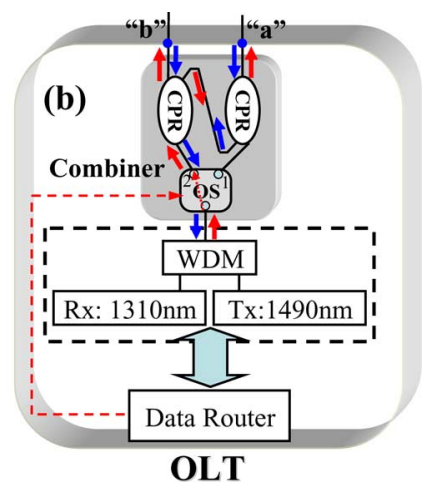

OLT
Fig. 2. Proposed combiner in OLT to control the transmitted direction of downstream signal, when the direction of OS is set to (a) the "1" point and (b) "2" point.

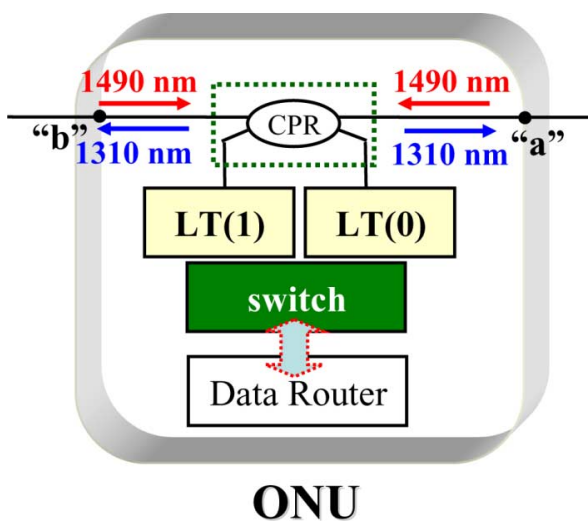

Fig. 3. Proposed ONU module with the bidirectional function.

The downstream signal of OLT is transmitted through the path "a" (clockwise) in Fig. 1 without any fiber failure (in normal state). As illustrated in Fig. 2, the OLT can determine two different transmission paths for data traffic by the proposed combiner integrating in OLT. The proposed combiner consists of two $1 \times 2$ optical couplers (CPRs) and a $1 \times 2$ optical switch (OS). The switching direction of OS can be controlled by the media access control (MAC) without an additional control system. In the normal state, the OS is placed at " 1 " point for the downstream traffic through the "a" fiber path, as shown in the left side of Fig. 2. Moreover, Fig. 3 presents the proposed ONU with bidirectional function to access the downstream and upstream links. The physical layer of each ONU is constructed by a $2 \times 2 \mathrm{CPR}$ and two LTs. The upstream traffic will be transmitted from the LT(1) through path "a" (counterclockwise) without any fiber failure. $\mathrm{LT}(0)$ of ONU is prepared against the fiber failure. In Fig. 1, when a fiber fault occurs between $\mathrm{ONU}_{2}$ and $\mathrm{ONU}_{3}$, then the data traffic of $\mathrm{ONU}_{3}$ and $\mathrm{ONU}_{4}$ cannot link with the OLT. At this time, the two unreachable ONUs will start driving the $\mathrm{LT}(0)$ s to reconnect the data links simultaneously in Fig. 3. And the OLT will switch the direction of OS to the "2" point by MAC, as seen in the right side of Fig. 2. Then, the downstream signal will be separated to pass through the "a" (clockwise) and "b" paths (counterclockwise) simultaneously for data traffic. As a result, the data links from OLT to $\mathrm{ONU}_{1}$ and $\mathrm{ONU}_{2}$ are routed through the "a" path (counterclockwise), and the $\mathrm{ONU}_{3}$ and $\mathrm{ONU}_{4}$ are routed through the "b" path

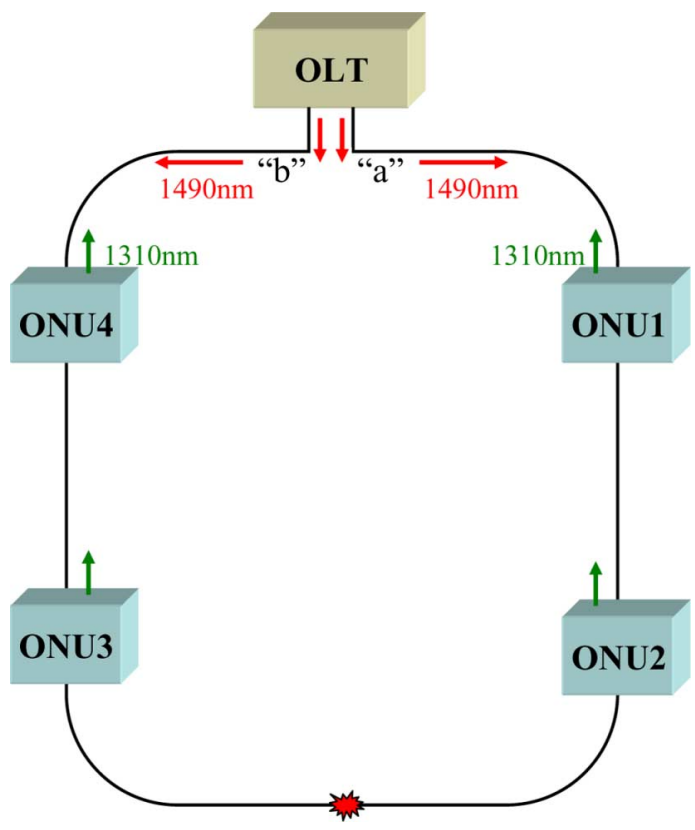

Fig. 4. Data traffic of proposed self-protecting ring-based PON with four ONUs when a fiber fault occurs between the $\mathrm{ONU}_{2}$ and $\mathrm{ONU}_{3}$.

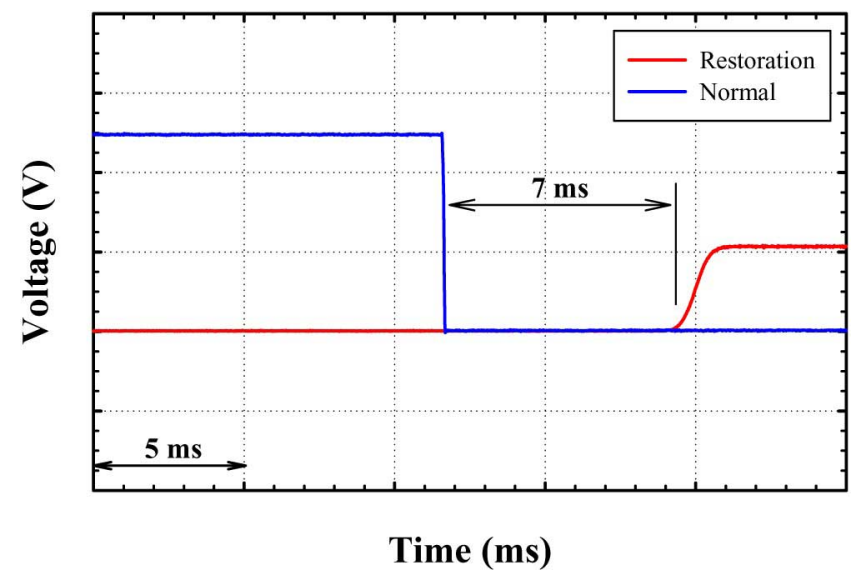

Fig. 5. Restoration of the proposed ring-based PON after fiber fault occurring. The restorable time is within $7 \mathrm{~ms}$.

(clockwise), as illustrated in Fig. 4. When the fiber failure is restored, then the operation mechanism of the PON system will be restored. Besides, before the transmission failure (normal state), this downstream signal was passed through the "a" path. However, in case of fiber cut, it is no longer possible to receive the upstream signals behind the fault point. Thus, this downstream signal is split and transmitted through the "a" and " $b$ " paths by switching the direction of OS to " 2 " point, simultaneously. This restorable time is achieved within $7 \mathrm{~ms}$ in the proposed access network, as shown in Fig. 5.

\section{EXPERIMENTS AND RESULTS}

To estimate the system performance of the proposed self-protecting ring-based PON, an experiment is performed. The experiment is setup in Fig. 1. A transmission distance between the OLT and $\mathrm{ONU}_{4}$ is $20 \mathrm{~km}$ long through the path "a". The 1490-nm downstream and 1310-nm upstream signals have 


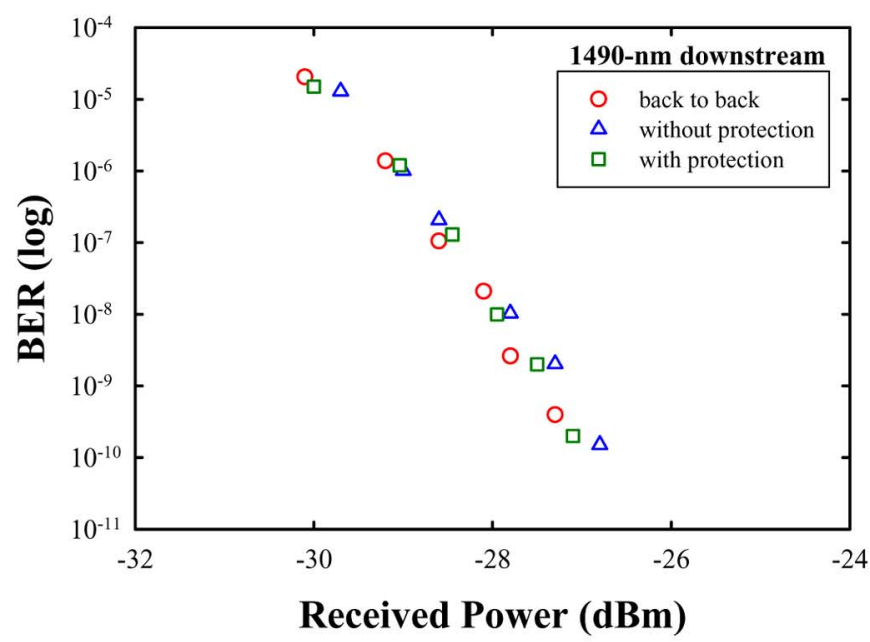

(a)

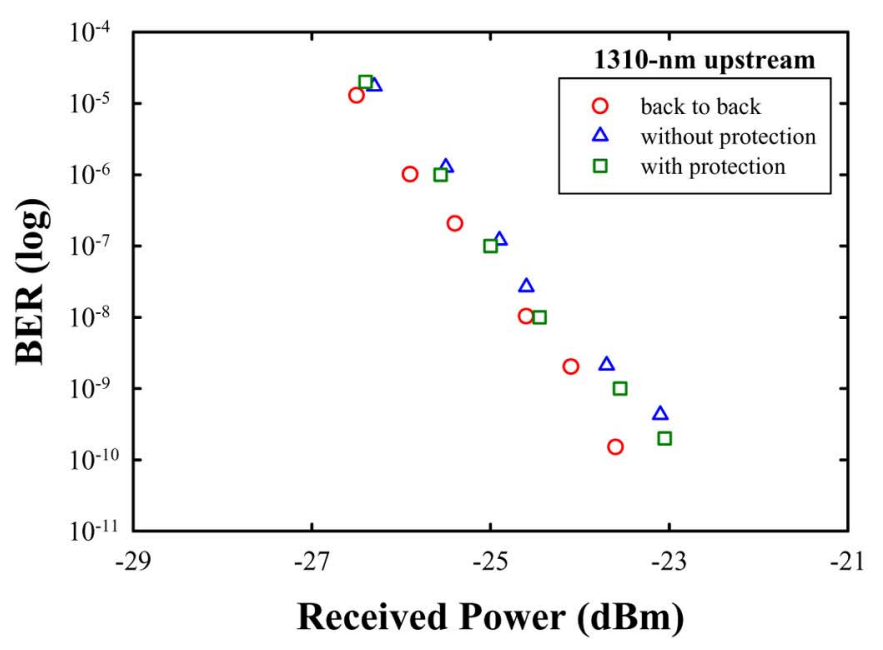

(b)

Fig. 6. BER performance of (a) downstream and (b) upstream traffic at $1.25-\mathrm{Gb} / \mathrm{s}$ modulation. The distance between the OLT and $\mathrm{ONU}_{4}$ is $20 \mathrm{~km}$ long.

$1.25-\mathrm{Gb} / \mathrm{s}$ direct modulation. In addition, in regard to system power budget, 1490- and 1310-nm signals will traverse an OS $(<1 \mathrm{~dB})$, five CPRs $(<16 \mathrm{~dB})$, and about 20 -km single-mode fiber ( $4 \mathrm{~dB}$ ); thus, the loss budget is about $<20 \mathrm{~dB}$. This scalability of the proposed ring network is nearly $26 \mathrm{~dB}$. The bit-error-rate (BER) performance is measured by a $1.25-\mathrm{Gb} / \mathrm{s}$ nonreturn-to-zero pseudorandom binary sequence with a pattern length of $2^{31}-1$ for the downstream and upstream traffic between the OLT and $\mathrm{ONU}_{4}$ through "a" (without protection) and "b" (with protection, fault between $\mathrm{ONU}_{3}$ and $\mathrm{ONU}_{4}$ ) paths. In the measurement system, the output power of 1490and $1310-\mathrm{nm}$ transmitters is 2 and $2.5 \mathrm{dBm}$, respectively. Fig. 6(a) and (b) shows the measured downstream and upstream BERs in the self-protected ring-based PON against the received power through "a" and "b" paths between OLT and $\mathrm{ONU}_{4}$. The observed optical power penalties are smaller than $\sim 0.2 \mathrm{~dB}$ in Fig. 6 while the BER is $10^{-9}$.

Simultaneously, to evaluate the feasibility of the proposed architecture, we also measure the throughput performance of the $1.25-\mathrm{Gb} / \mathrm{s}$ downstream and upstream traffic by directly connecting a performance analyzer with a frame length of 1518 byte. The throughput performance of downstream and upstream traffic is $98.59 \%$ and $99.28 \%$ in the proposed access network, respectively.

\section{CONCLUSION}

We have proposed and investigated a new ring-based time-shared PON with self-healing function to prevent the fiber-fault occurring. By using the new optical line terminal (OLT) and single fiber path architecture, the proposed self-healing ring topology PON can be revived promptly under single fiber failure. The access system performances are also measured and discussed in the proposed architecture.

\section{ACKNOWLEDGMENT}

The authors would like to thank C.-S. Lee and S.-L. Yeh for help with the experiments.

\section{REFERENCES}

[1] Broadband Optical Access Systems Based on Passive Optical Network (PON), ITU-T Recommendation G. 983.5, 2002.

[2] Gigabit-Capable Passive Optical Network (GPON): General Characteristics, ITU-T Recommendation G. 984.1, 2003.

[3] Ethernet in the First Mile Task Force, IEEE 802.3ah, Draft 3.0b, 2004.

[4] T. J. Chan, C. K. Chan, L. K. Chen, and F. Tong, "A self-protected architecture for wavelength-division-multiplexed passive optical network," IEEE Photon. Technol. Lett., vol. 15, no. 11, pp. 1660-1662, Nov. 2003.

[5] K. D. Langer, J. Grubor, and K. Habel, "Promising evolution paths for passive optical access networks," in ICTON'04, 2004, pp. 202-207. 